\title{
COGNITIVE MODELLING FOR ENGINEERS
}

\author{
William F. Gaughran \\ Department of Manufacturing and Operations Engineering \\ University of Limerick
}

\begin{abstract}
Computer modelling software endeavours to generate technically accurate and sometimes photo-realistic models of design intent. A significant time investment is devoted to becoming proficient in the use of one or more CAD software and other modelling packages, in the design, production and modification of products for design and manufacture. The creative use of CAD software, depends greatly on the users cognitive ability to visualise the design intent and to interact with the developing model of the product. The research shows that cognitive modelling capacity is not an innate skill of the engineer but that it can be developed by appropriate intervention strategies. At the University of Limerick, interactive tutorial software has been developed to improve the users spatial modelling capacity. It works in tandem with other intervention strategies. The results show significant improvements in the users spatial ability and visualisation skills - resulting in greater creativity and more productive use of CAD modelling systems as a design tool. Improved cognitive modelling skills, enables the engineer to perform on a higher creative plane in most areas of engineering activity. $>\quad$ Key Words: Design Intent, Visualisation, Spatial Reasoning, Creativity
\end{abstract}

\section{Designing and Visualisation}

Improving design, manufacturing and production efficiency has for obvious reasons been a focal point of engineers for some time. Engineers are now very much part of the design team working together to improve efficiency. Most design teams work to a system which impacts on all facets, from concept to consumer and in the light of 'green' issues, design for sustainability is also becoming a very important a part of the strategy. This approach has given rise to manufacturing strategies such as 'total design', 'concurrent engineering' and more recently 'collaborative design and engineering'. In 'total design' all the production team co-operate to produce the best and most cost-effective solution to design problems. All, are concerned with improving, modifying and quality controlling at each phase of production and distribution. All team members are familiar with the source and manufacture of the basic components as well as where and how they will be used in the next and subsequent phases of the product's development. Not all members of the team involved in product design, development and manufacture will be engineers, however all the engineers will be involved in some way in designing. 
It may be said that design is at the core of all facets of engineering. There are many definitions of design but Blumrich's, in Deiter $1991^{1}$ description would appear to fit well the design activities of most engineers; 'Design establishes and defines solutions to and pertinent structures for problems not solved before, or new solutions to problems which have previously been solved in a different way'. This definition shows that design reflects the creative and sometimes the innovative activities of the engineer. It also gives quite a broad brief to the engineer. Regardless of how one defines engineering design it is a creative activity and the essence of creativity is visualisation. This however is a cognitive capacity which many engineers lack and many may even see as unimportant. Visualising, being creative and designing, in the context of engineering, are normally cognitive functions of the right cerebral hemisphere.

\section{The Split Brain and Lateralisation}

The human brain is divided into two halves sitting side-by-side and interconnected by the corpus callosum. The left cerebral hemisphere is normally identified with language and mathematical logic while the right is generally seen as the part which, creates images, visualises, designs and is creative. These specialisms are basically what lateralisation is about. Many do not recognise this as having any great significance to the engineer. However if one analyses the attitudes we have and the language which we use, we will quickly see that in an off-hand way, lateralisation or cerebral specialisms has been recognised for centuries. It is well recognised that the left hemisphere of the brain controls the right hand and visa-versa for the left hand (Figure 1).

The left hemisphere of the brain controls the right side of the body

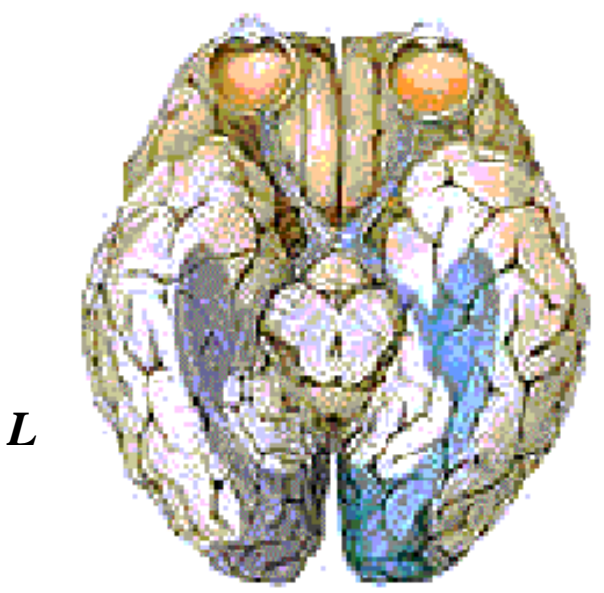

The right hemisphere of the brain controls the left side of the body

Figure 1 - Right/Left crossover in control and processing

'Left' and 'right' have their own connotations in every day language. In the not too distant past many young people who were naturally left-handed were forced to change to right- hand use, for example in writing. Not too many centuries ago being lefthanded was seen as sufficient reason to be burnt at the stake. Do such attitudes still 
exist, even in a subtle way? Being 'right' has to do with 'correctness', 'good', 'proper' and so on, but being left or left-of-centre is completely the opposite.

To illustrate: A left-handed (right-brain dominant) person is termed a sinistral. This word derives from the Latin for left, which is 'sinister'. On examination of the Oxford Thesaurus, 1991, the synonyms for sinister include; evil, bad, corrupt, sneaky, underhanded, harmful and many others. A right-handed person is termed a dextral; this word derives from the Latin 'dexter'. Synonyms for dexterity are; skill, proficiency, deftness, clever, keen and so on. This bias appears in all common languages. The French word for left is gauche, which means 'awkward' (from gawk or gallock-hand, left-hand). The French for right is droit, from 'good', 'just' or 'proper'. In Irish a lefthanded person is called a ciot6g; which implies helpless, awkward, contrary. The Irish for a right-handed person is deasach from 'deas' - meaning 'appropriate', 'attractive', 'clever', 'witty'. In English, left comes from the Anglo Saxon lyft, meaning 'weak' or 'worthless'. Whereas Anglo-Saxon reht meant 'straight' or 'just', (Edwards, 1989) ${ }^{2}$, The Latin word associated with reht is rectus from which we derive 'rectitude' or' correct'.

All the foregoing illustrate the negativity associated with left-handedness, which indirectly indicates (although sometimes unwittingly) the concentration on left-brain development, at the expense of the right hemisphere. The prejudice is still found in psychological research where some refuse to recognise left-handers and prefer instead to refer to this group as 'non-right-handers'.

Why though is it important to focus greater energy on developing the right cerebral hemisphere, particularly in engineers?

\section{Visualisation and Lateralisation}

It is widely accepted that the right cerebral hemisphere is responsible for processing design and spatial details and for the cognitive manipulation of such data. An important ability for any engineer is that of 'cognitive modelling'. This involves building and manipulating images so as to define, refine and communicate ideas and solutions to design and engineering problems. Visualisation while used as a general term for building cognitive images or models is also seen as a subset of a more general human intelligence, namely, 'spatial ability'. Lohman's, $1979^{3}$ definition of spatial ability is; 'the ability to generate, retain and transform abstract visual images' and Gaughran, $\left(1996^{4,5}, 2000^{6}\right)$; 'the ability to visualise, manipulate and interrelate real or imaginary configurations in space'.

While researchers in the past have identified two or three sub-factors to spatial ability, Gaughran, $\left(1990^{7}, 1996^{4}\right)$ recommends five sub-factors. Before these are discussed we will first contextualise spatial ability in relation to the overall human intelligence factor ' $g^{\prime}$. Vernon $\left(1961^{8}\right)$ and Smith $\left(1964^{9}\right)$ identify two major subsets to the general intelligence factor ' $g$ '; these are the ' $V: e d$ ' group and the ' $k: m^{\prime}$ group. Where $V: e d$ represents the language/logical-mathematical grouping of intelligence factors and $k: m$ 
represents the visuo-spatial/psychomotor intelligence factors. The former is normally a left-brain function and the latter a right-brain activity. See Figure 2.

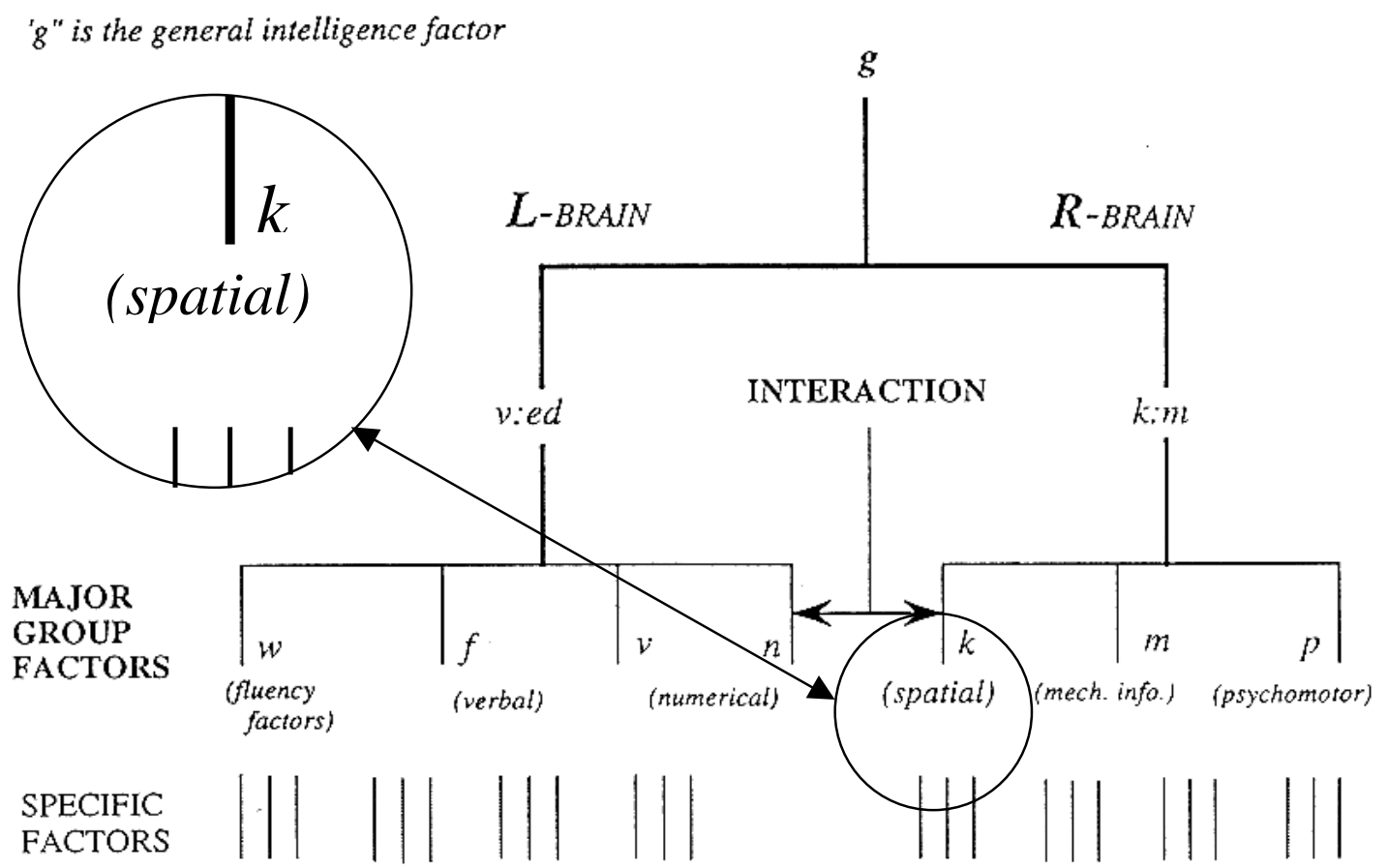

HIERARCHICAL STRUCTURE OF HUMAN ABILITIES

Figure 2

Each of the sub-groups has their own sub-factors, and to have satisfactory methods of developing these, they must be defined and categorised. Some of the sub-factors of spatial ability identified in the past have been confusing to say the least; for example what Zimmerman $(1954)^{10}$, describes as 'spatialising', French $(1951)^{11}$ appears to call 'orientation'. Several others, including French uses the term 'Visualisation' to apply to a single sub-factor of spatial ability. The general definition of visualisation: 'to form a mental image', would appropriately apply to all sub-factors of spatial ability. In order to better develop meaningful tutorial intervention strategies, Gaughran $(1990 / 96)^{6,4}$ suggested expressing the sub space-factors (SF) in ascending order, according to factorof-difficulty, viz.

(SF-1) Image Holding and Comparing: Involves spatial decomposition and observing topographical relationships. An example would be to build an image of an electric plug-top and compare its topology with a similar one.

(SF-2) Planar Rotation: Of two or three-dimensional objects. An example would be to rotate the plug-top on a single plane (the vertical or horizontal).

(SF-3) Spatial Orientation: The observer will move to occupy various imaginary vantage points, while the object remains static, e.g. instead of mentally rotating the plug-top as in SF-2 to acquire a different view, the 'imager' imagines viewing it from different orientations. 
(SF-4) Kinetic Imagery: Imagining an object as it changes position in space moving in any axis, e.g. the ability to manipulate the image of the plug-top in any axis in space.

(SF -5) Dynamic Imagery: The ability to manipulate elements within an object this would include the ability to 'explode' and re-assemble the components of the plug-top. See Figure 3.

An electric plug-top can illustrate the cognitive application of Gaughran's five sub-factors

\section{Figure 3}

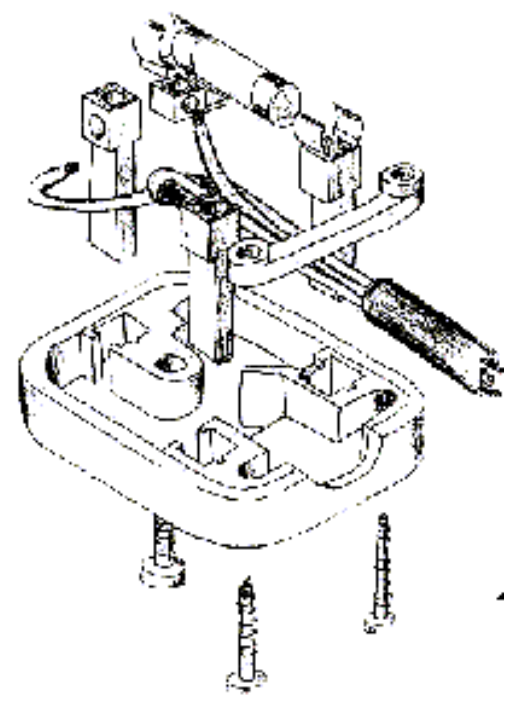

While $S F-I$ is a pre-requisite of all other sub-factors, the other space factor may use one, more or as in the case of $S F-5$ all the others. Each builds on the other downwards (Fig. 4). Planar rotation and orientation may interchange or combine in the cognitive manipulation of an image. The logical left-brain- may also cooperate, using mathematical algorithms to check size, fit, etc. This is the ideal dual-processing cognitive activity.

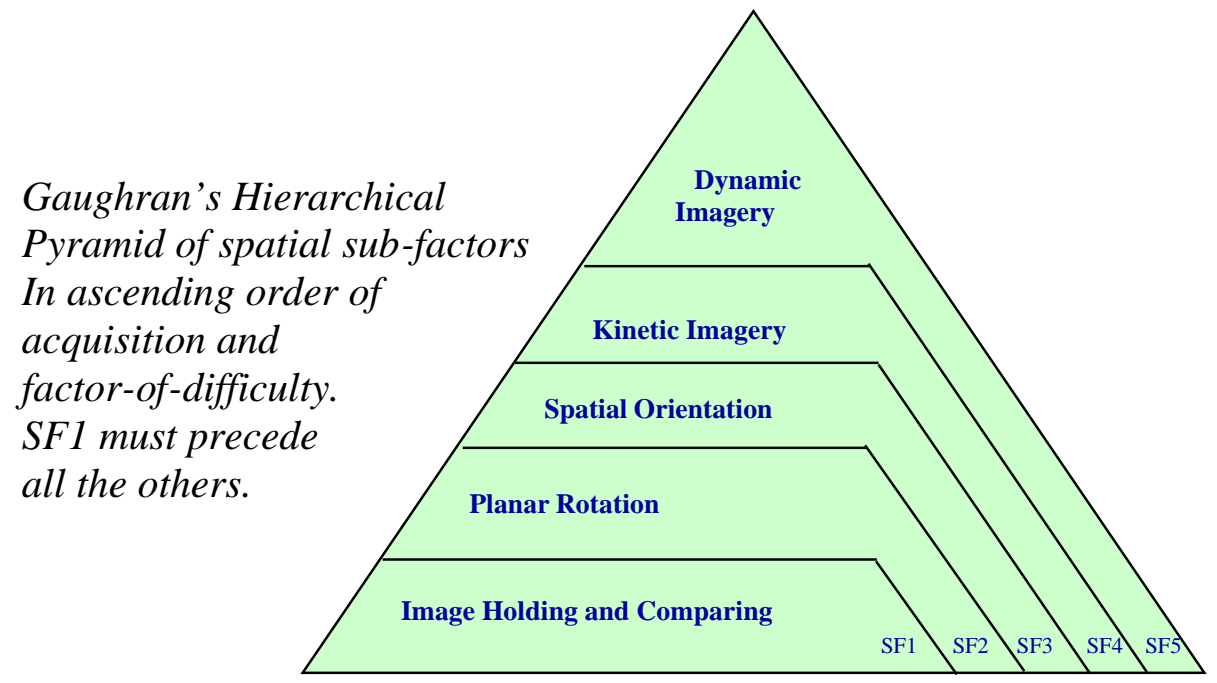

Figure 4 - Gaughran's Pyramid of Re-defined Spatial Sub-factors (SF1 to SF5) 


\section{Strategies that Work}

Some psychologists say that spatial ability is innate and that laterali sation of cerebral functions has been decided at birth. However where appropriate tutorial intervention is employed significant advances can be achieved regardless of age, (Lord ${ }^{12,13}$, Moses ${ }^{14}$, Gaughran ${ }^{4,5}$ ). In order for tutorial intervention to be effective cognizance must be taken of individuals with quite limited spatial experience. A sequenced approach is advisable, for example Mamaw and Pilligrino ${ }^{15}$, Figure 5.
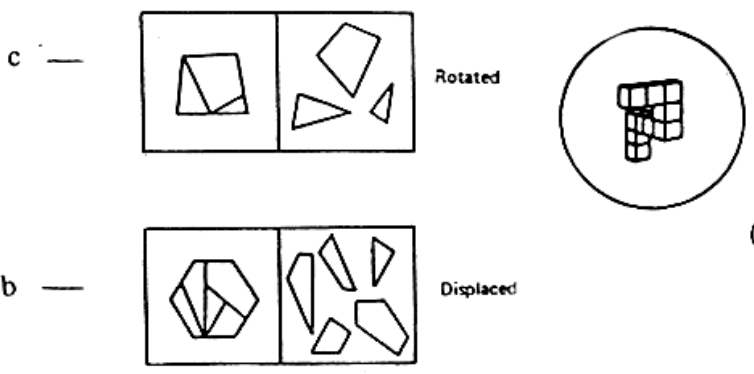

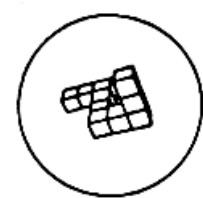

(a)
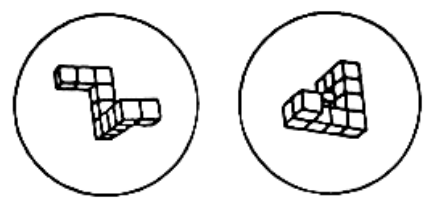

(b)

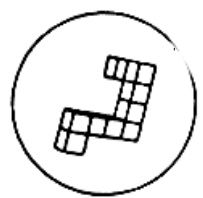

Figure 6 (c)

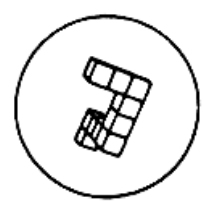

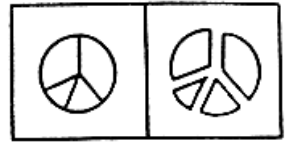

Figure 5

Considering the speed of perception in relation to the angle of rotation, Shepard and Metzler ${ }^{16}$ found when using cubular arrays in perspective that the average rate of rotation is approximately $60^{\circ}$ per second, see Figure 6 .

They (Shepard and Metzler) presented perspective view in three different ways: (a) pair that differs by an $80^{\circ}$ rotation within the picture plane; (b) differs by rotation in depth and (c) cannot be brought into congruence by any rotation, they are enantiomorphic (mirror images). The mirror image causes difficulty for most learners due to cognitive confusion with left-right orientation. Mirror imaging is however very useful in the design of distracters for spatial testing. These examples illustrate how a progressive strategy may be employed to increase the factor-of-difficulty and how in threedimensions the process of encoding, comparison, search, rotation and decision can be followed. The individual with a more advanced spatial ability will more quickly reduce the set of alternatives in processing correct solutions.

The above may be supplemented with models and tactile stimuli. Understanding the cognitive process, or at least its sequence, assists in designing meaningful tutorial intervention strategies. The gradual rotation of sectioned elements assists the beginner and accommodates the more advanced in arriving at an early conclusion. This method provides the learner, with a very useful representational 'Bridge', i.e. it eases the learner through the mental rotation steps which is how the cognitive model is rotated, see Figure $7^{17}$. Here again a physical model which is sectioned and gradually separated will 
assist the beginner and reinforce the learning, through tactile as well as visual sensory input. It has also been observed that the act of testing spatial ability, will in itself stimulate improvement in the spatial performance of the subject in subsequent testing.
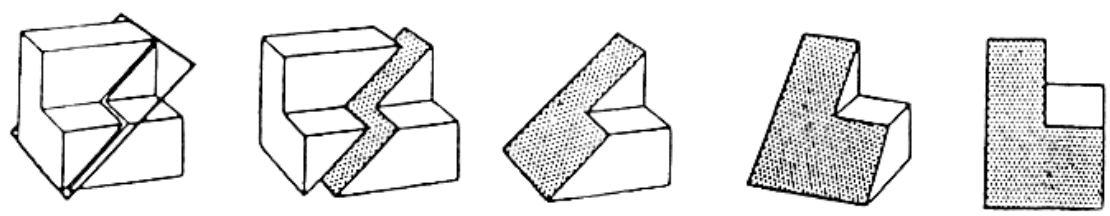

Eliot and $\mathrm{Smith}^{17}$
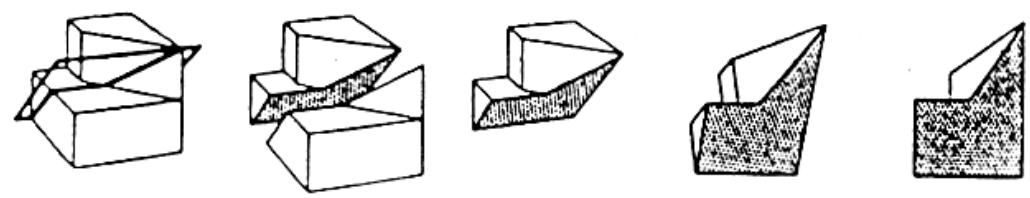

Figure 7

The first graphic shows the cutting plane penetrating the solid and gradually the cut section is rotated towards the viewer. It appears that $10^{\circ}$ is a good rotational interval (Stepsize). They (Seddon, et al) ${ }^{18}$ observed that students performance decreased significantly as the stepsize increased. In improving visualisation skills the ability to identify and respond to depth and size cues is an essential pre-requisite. An example is seen in Figure 8.

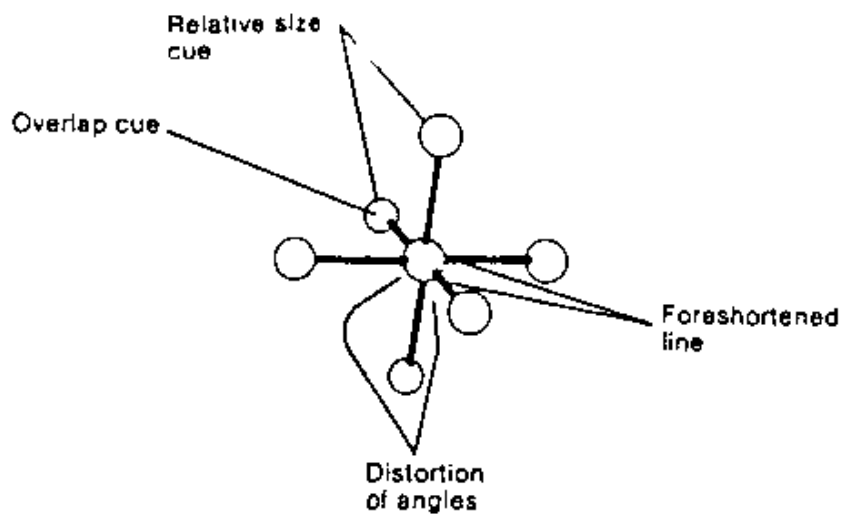

(In the actuai model being represented alt the spheres are the same size;
all the rods are the same length, and all the angles between adjacent rods are $\left.80^{\circ}\right)$.

Figure 8 - examples of four different depth and size cues

The designing of tutorial media is complex in that it requires taking cognizance of the variety of intellectual levels and the type of cognitive processes involved. Gaughran ${ }^{6}$, designed and tested a series of computer based tutorials, taking cognizance of pre vious findings and applying the concept of the five sub-factors, to determine whether this mode was effective. The graphics elements were mainly 3D and fully interactive. 
When the experimental group, were compared with the control group there were significant increases in spatial ability in all elements SFI to SF4. SF-5 was not tested on this occasion, because of its complexity and would require much more extended tutorial intervention. The tests and tutorials were carried out over a three-week period. The post-testing of the experimental and control groups was conducted three to four days after the tutorials. While the positive results confirm that there is cerebral specialisation in connection with visuo-spatial processing, the significant evidence of cerebral lateralisation is quite interesting.

A cohort of about 120 students were involved of which $12.5 \%$ were left-handed. The complete cohort undertook the same pre-test and were later divided for tutorial sessions. They were given exactly the same series of tests on the post-test and the improved score of the control half of the cohort was used as the 'familiarity-factor' and subtracted from the raw scores of the experimental group. This produced an average improvement in score of just under $7 \%$ for the experimental group and this, when subjected to statistical analyses proved to be significant.

\section{Points of note:}

$>\quad$ In the pre-test the sinistrals scored $12 \%$ better than the dextrals

$>\quad$ In the post-test the sinistral's score improvement was nearly double that of the dextrals.

$>$ The top seven scores in the experimental cohort scored above $75 \%$ - three of these $(43 \%)$ were sinistrals.

In 1988 a group of children were tested so as to determine whether visually gifted children retain visual images without the intention of deliberately committing them to memory. The gifted children were better able to identify the altered picture and also recalled more of the specific details. The researchers were surprised to find that, $24 \%$ of the gifted children were left-handed and that none of the non-gifted children were lefthanded - (Resenblatt and Winner, 1988) ${ }^{19}$.

Recent studies at the University of Limerick on whether previous drawing experience affects the computer aided design scores of undergraduates are also interesting. In one group tested there was a $17 \%$ difference in scores by those students with previous advanced drawing experience. The results at the top of the group were particularly interesting. Seven of the top eight performers had additional drawing experience. The eight member of this cohort had an 'A' in higher level mathematics and he was lefthanded. Two of the top five, were left-handed (sinistrals constituted $12 \%$ of the cohort of 75 students).

The foregoing is not intending to say that we need more left-handed engineers but it does illustrate that where handiness is considered it is an indicator of cerebral specialism and lateralisation. It is also worth noting that sinistrals are less specialised cerebrally. It is estimated that while $99 \%$ of dextrals have language processing in the left-hemisphere and that only $66 \%$ of sinistrals have left-hemispherical language processing. This shows that there is less cerebral specialism among sinistrals. This is supported by research on left-handers by Witelson $(1985)^{20}$. Witelson found that on 
average the corpus callosum in the brain of sinstrals to be an average of $11 \%$ larger than in dextrals.

\section{Drawing and Spatial Intelligence}

To what extent does drawing/graphics experience give a spatial advantage? One researcher found evidence that : individuals who have lower visualisation skills will perform essentially at the knowledge and comprehension levels, while those individuals who have developed more enhanced visualisation skills will perform more consistently at higher levels of conceptualisation and reasoning". (Tally, 1973) ${ }^{21}$. Steve Garner observed that: "In spite of the breadth of case studies there appears to be unanimous support for the importance of drawing (both measured and freehand). Not only is it seen as an appropriate means of defining a better solution or product but it is presented as profoundly affecting the processes (Garner, 1992) ${ }^{22}$.

As part of an interactive process of drawing and thinking or 'cognitive modelling' some use the term 'homing in'. This expands the role of drawing beyond being a problemsolving device, to being a 'problem-finding' one. One well-known designer stated that the "whole process of drawing is to do with identifying very clearly what the problem actually is". A well arranged drawing or sometimes a quick sketch clearly defines the problem in its interaction with the thought process, which has generated the cognitive model. The drawing in this sense is not just the model of the idea, it often is the idea.

Anita Cross in focusing on a design intelligence also describes a 'graphic intelligence'. Referring to the work of Van Sommers, she writes that: "competent drawers often employ learned strategies. But, when these strategies are inadequate or lacking, a type of graphic intelligence is exercised to solve new problems of perceptual analysis posed by complex subject matter" (Cross, 1986) ${ }^{23 .}$

Van Sommers (inCross ${ }^{23}$ ) studies reveal that : "successful drawers tend to use successful strategies". Cross, describes drawing as: "a descriptive code containing particular types of spatial concepts not contained in verbal language". It has been found that this graphic intelligence involves engaging in the meanings and semantics of a given task. Cross further states that: "these meanings are released by the act of drawing, thus, the act of drawing makes meanings accessible to conscious attention for focusing".

If we recognise that drawing has a unique descriptive code that can only be acquired efficiently by the act of drawing, then we can begin to prepare people to exploit the full potential of the engineer in creative activity. Engineers, before and during CAD activities will then begin to employ the cognitive process of 'graphic ideation', which is described as: "the activity of using drawing or sketching to assist the thinking process" (Mc Kim, 1980) ${ }^{24}$. The sketch can therefore be seen as the externalization of the cognitive model, the first visible evidence of the idea or solution. The sketch which assists and clarifies the thought process, might in its init ial stages, require to be vague. This prevents the designer or problem solver from focusing too early on a solution or 
design details. Graphic ideation becomes a vital part of the cognitive modelling process and the creative activity of the engineer. Figure 9, shows how Da Vinci used vague concept drawings, in the design of his Flying Machine. We can see from his earlier and later sequenced sketches how he employed 'graphic ideation' in the process.

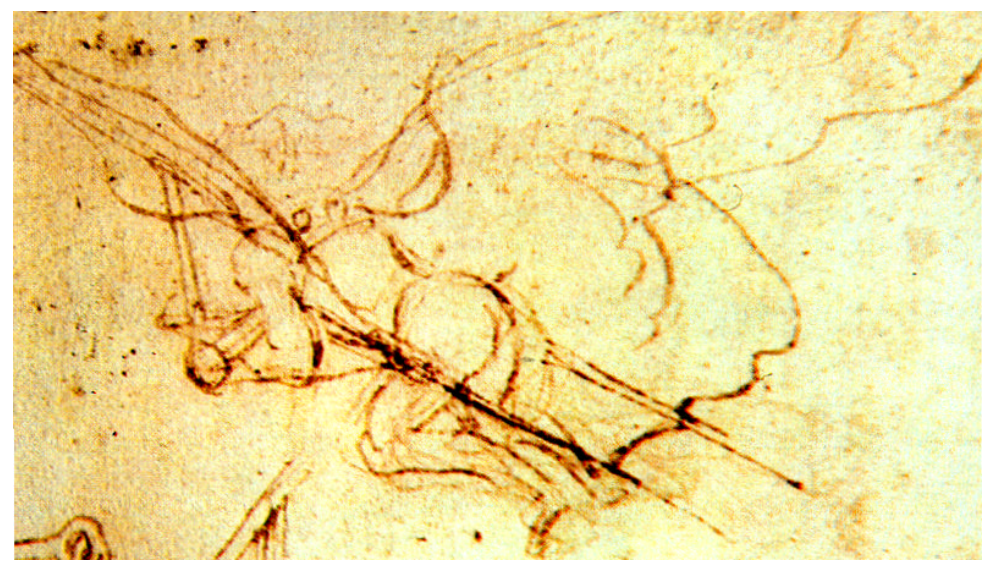

\section{Figure 9}

One of Da Vinci's 'graphic ideation' sketches for the Flying Man.

Recently developed computer based tutorials at UL have the advantage of rendering and animation in demonstrating the spatial sub-factors. These tutorials are interactive and as well as progressing vertically through Gaughran's hierarchical pyramid, each sub-factor is addressed at three levels; beginner, intermediate and advanced (see Fig. 10). The tutorials work in conjunction with a computer based test bank, which gives immediate feedback to the spatial learner regarding levels of progress.
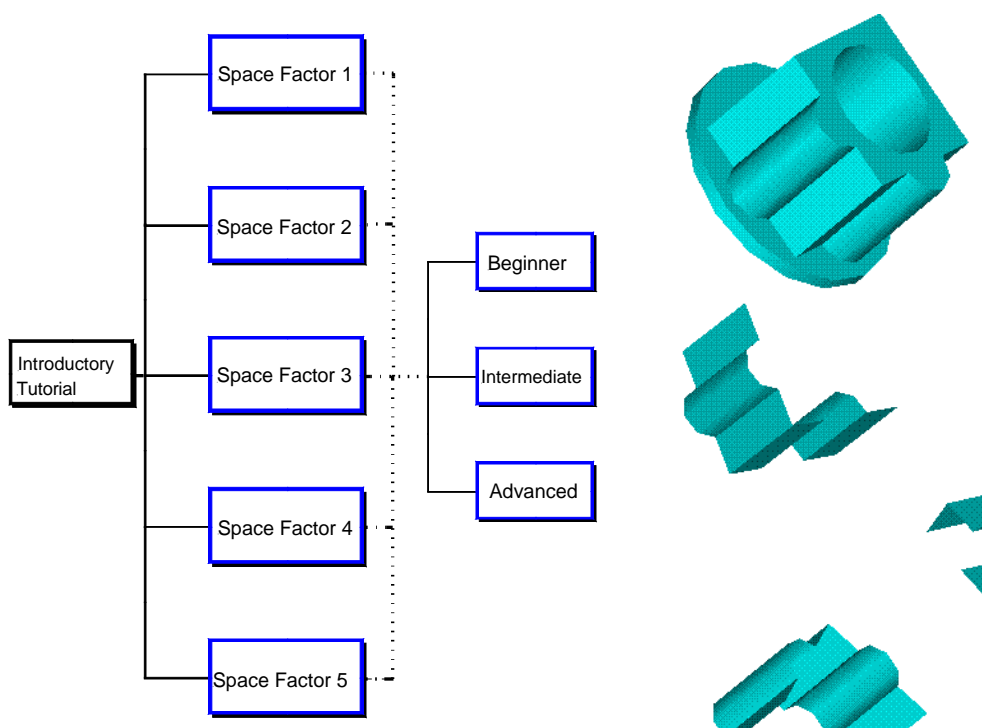

Which of the five objects is a fragment of the surface of the test object?
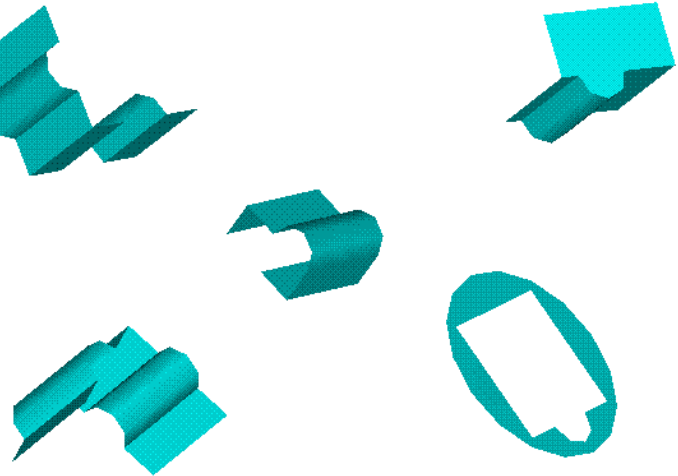

Figure 10 - flow chart and one element, where the fragment is animated onto the object

A bank of computer based spatial tests, which also addresses the sub-factors has also been developed. The test bank gives feedback scores for each of the sub-sections as well as overall scores. Each sub-section has elements, which progressively increase in 
factor-of-difficulty and which, while testing also give a spatial experience to the subject that indirectly improve subsequent spatial performance. An example of one such element, relating to sub-factor 1, is shown in Figure 11. The subject is first shown one element and then asked to identify the correct element from the five (one correct and four distracters).
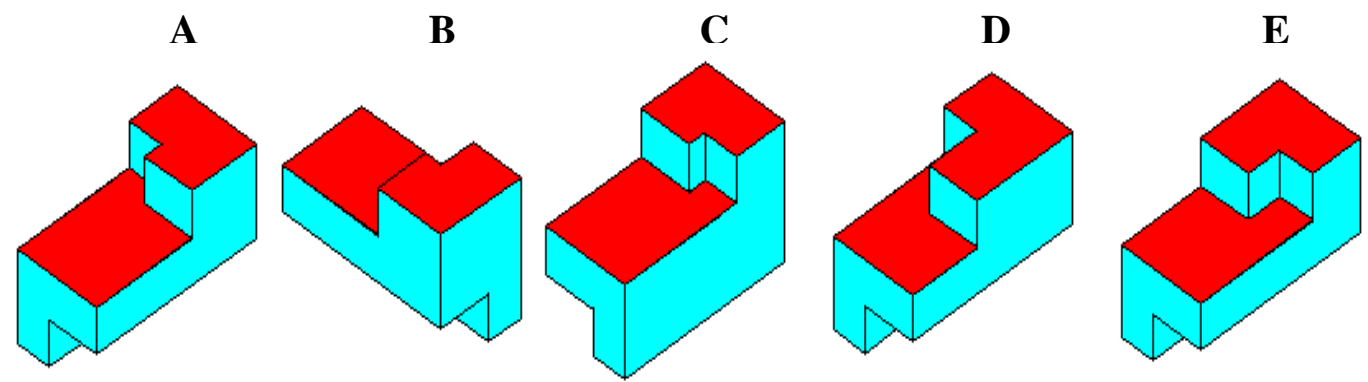

Figure 11 - Example of computer based test element for SF1

A similar example is shown for sub-factor 5 in Figure 12, where the pieces must be dynamically maneuvered to assemble the correct pieces to represent a given model.

Select the three elements which will combine to build the model

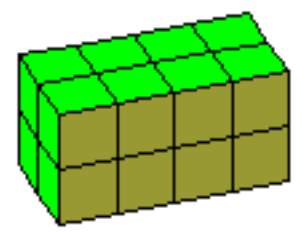

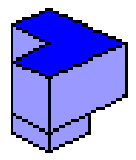

A

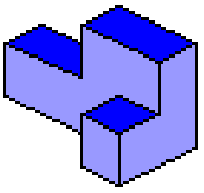

B

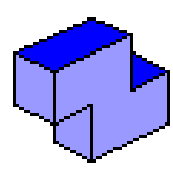

C

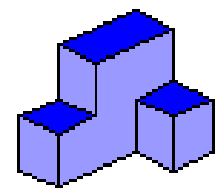

D

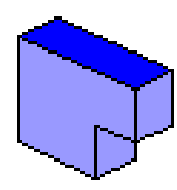

$\mathbf{E}$

Figure 12 - Example of computer based test element for SF5

Along with specifically designed tutorial programmes, the advent of advanced methods of computer model display using Java and other visualisation methods such as eDrawings (see Figure 13) allow visual interrogation of models and components. These, along with tactile and purpose designed tutorial material which takes into account the processing of spatial information, including the recognition of lateralised processing allow the designers of such programmes to make appropriate tutorial intervention. 


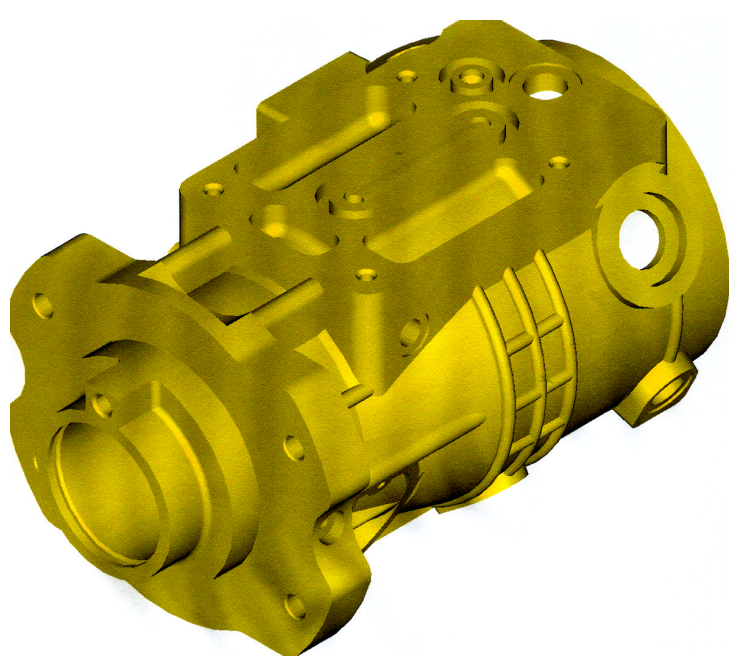

eDrawing from SolidWorks

Can be rotated, zoomed, animated, seen as an engineering ortho view shaded for better visualization and other useful functions - without running the programme. Only the viewer is required. This interaction has obvious benefits in cognitive modelling

Rapid prototyping is rightly seen as an important tool in compressing the time from concept to production in product or component design - the cognitive model which an engineer or designer, with a developed level of visualisation skills can allow for greater accuracy in prototyping. Taking time to develop the ability to build cognitive prototypes makes for more creative engineering and makes good economic sense. The tutorial interventions discussed here will contribute to the reengineering of the engineer whether in the undergraduate stage of development or as a practitioner in any field of engineering.

\section{Discussion}

Lateralisation involves the two hemispheres in different aspects of cognitive processing. In order to improve engineering performance, cognizance should be taken of the necessity to stimulate right-brain activities such as holistic reasoning and image building or cognitive modelling and the important human intelligence of spatial ability. While many contend that individuals either possess this ability or they don't (that it is innate) research shows that with appropriate tutorial intervention, this ability can be developed and enhanced. The previous and ongoing research at the University of Limerick demonstrates the effectiveness of such intervention. The design and inclusion, of a revised test-bank, pre-CAD tutorials and tactile stimuli are showing promising initial results and will add significantly to the effectiveness of the overall strategy.

Engineers are constantly involved in design activity and need to be good visualisers. They should to be able to build the most cost-effective of all rapid-prototypes - the cognitive model. This can only be achieved by recognition of the need to 'spatialise' design/engineering information. The recognition of cerebral lateralisation should influence the design of undergraduate courses for engineers and later their in-career development, and assist in making them more creative and innovative problem solvers.

"The left hemisphere analyses over time whereas the right hemisphere synthesises over space " - (Levy, 1974) $)^{25}$. 


\section{References}

1. Deiter, G.E., 1991, Engineering Design, McGraw Hill, Boynes 1978.

2. Edwards, B., 1979 and 1993, Drawing on the Right Side of the Brain, Collins and Sons, Glasgow.

3. Lohman, P.F., 1979, Spatial Ability: A review and reanalysis of the Correlation Literature, Technical Report No. 8, Stanford University

4. Gaughran, W.F. 1996, The Graphics Code Visualisation and CAD, FAIM Conference, Atlanta May'96.

5. Gaughran. W.F. 1996, Design Intelligence on Engineering, Paper to the IMC- 13 Conference, Limerick Sept. 1996.

6. Gaughran, W.F., 2000 . Modelling and Design Intent, Engineering and Product Design Conference, University of Sussex, England, Sept. 2000

7. Gaughran, W.F. 1990, Developing Spatial Abilities Through Computer Assisted Learning, M.Tech. Thesis, University of Limerick 1990

8. Vernon P.E., 1961, The Structure of Human Abilities, Methuen, London..

9. Smith, I.M., 1964, Spatial Ability - Its Educational and Social Significance. N.F.E. R., Nelson, Berks.

10. Zimmerman W.S., 1954, Hypotheses Concerning the Nature of Spatial Factors Education and Physical Measurement Vol. 14, 306-400

11. French, M.S., 1951, Conceptual Design for Engineers, Design Council London.

12. Lord, T.R., 1987, Spatial Teaching. The Science Teacher, February, 1987

13. Lord, T.R., 1984, A Plea for Right Brain Usage. Journal of Research in Science Teaching, 22, V, 395-405.

14. Moses, B., 1982, Visualisation - A Different Approach to Problem-solving, School Science and Mathematics, 82-2, 141-147

15. Mamaw R.J. and Pellegrino J.W., 1984, Individual Differences in Complex Spatial Processing, Journal of Ed. Psychology, V76, 5, 920-939

16. Shepard R.N. And Metzler J., 1971, Mental Rotation of Three-Dimensional Objects Science, Vol. 171, Feb.'71

17. Eliot and Smith J.M., 1983, An International Dictionary of Spatial Tests NFEF Nelson, Berks.

18. Seddon G.M., Eniaiyeju P.A. and Josoh J., 1984, The Visualization of Rotation in Diagrams of Three Dimensional Structure American Ed. Research Journal, Spring '89, 21, 1, 25 -38

19. Rosenblatt, E. and Winner, E., 1988 The Art of Children's Drawings. 18. Journal of Aesthetic Education, Vol.22, No. 1, Spring, 1988.

20. Witelson, 1985, in Graham R.B. 1990, Physiological Psychology, Wadsworth

21. Talley, L.H., 1973, The Use Of Three-Dimensional Visualisation: as a moderator in higher cognitive learning in College level chemistry.

22. Garner S., 1992, 'The Undervalued Role of Drawing in Design', in Thistlewood D (Ed), Drawing Research and Development, Longmans, London, pp 98-109.

23. Cross, A., 1986, Design Intelligence: the use of codes and language systems in design, Design Studies, Vol. 7, No. 1, January 1986.

24. McKim, R.H., 1980, Experiences in Visual Thinking, BWS Engineering, Boston

25. Levy, J., 1976, Cerebral Lateralization and Spatial Ability. Behaviour Genetics, T, No. 2, 171-188

William Gaughran is a Course Director in Technology Education and Lecturer in Engineering Design at the University of Limerick. He is Research Project Manager for inclusive design for facilities and tooling. He is a consultant and researcher to the National Council for Curriculum and Assessment (Ireland) and is the Author of several textbooks in technology education. His research interests include, inclusive/universal design, design for sustainability and human factors and cognitive strategies in design. 